\title{
GRAU DE DEPENDÊNCIA DE IDOSOS HOSPITALIZADOS PARA REALIZAÇÃO DAS ATIVIDADES BÁSICAS DA VIDA DIÁRIA ${ }^{1}$
}

\author{
Hellen Cristina Sthal ${ }^{2}$, Heloisa Wey Berti ${ }^{3}$, Valéria de Castilho Palhares ${ }^{4}$
}

\footnotetext{
${ }^{1}$ Recorte de projeto de pesquisa intitulado - Identificação da vulnerabilidade de idosos internados em enfermaria de ProntoSocorro de Hospital Universitário, desenvolvido com o apoio financeiro da Fundação de Amparo à Pesquisa do Estado de São Paulo, 2009.

${ }^{2}$ Pós-graduanda do Programa de Aprimoramento Profissional em Enfermagem em Saúde Pública da Faculdade de Medicina de Botucatu. São Paulo, Brasil. E-mail: hellen_sthal@hotmail.com

${ }^{3}$ Doutora em Saúde Pública. Docente do Departamento de Enfermagem da Faculdade de Medicina de Botucatu. São Paulo, Brasil. E-mail: weybe@fmb.unesp.br

${ }^{4}$ Mestre em Enfermagem. Supervisora da Seção Técnica de Enfermaria de Adultos do Pronto-Socorro do Hospital das Clínicas de Botucatu. São Paulo, Brasil. E-mail: valeria@fmb.unesp.br
}

RESUMO: Este estudo objetivou identificar o grau de dependência de idosos hospitalizados para a realização das Atividades Básicas da Vida Diária. Trata-se de estudo quantitativo, transversal e descritivo, desenvolvido na Enfermaria de Adultos do Pronto-Socorro do Hospital das Clínicas de Botucatu. A coleta de dados ocorreu mediante entrevistas, observação e consulta nos prontuários individuais. Os dados foram analisados com pacote estatístico SAS for Windows, versão 9.2, utilizando regressão logística. A amostra foi constituída por 71 sujeitos, com idade média de 74,30 anos. Verificou-se que 30,99\% dos idosos se apresentaram independentes na realização das atividades propostas, 22,53\%, parcialmente dependentes e $46,48 \%$ totalmente dependentes. O cuidado em gerontologia deve estar voltado para a manutenção do estado funcional, promovendo-se a independência e preservando a dignidade dos sujeitos.

DESCRITORES: Enfermagem. Saúde do idoso. Avaliação geriátrica. Atividades cotidianas.

\section{DEPENDENCY LEVELS IN HOSPITALIZED ELDERS IN PERFORMING BASIC DAILY LIVING ACTIVITIES}

\begin{abstract}
This study aimed to identify the level of dependence in elderly inpatients when performing Basic Activities of Daily Living. This quantitative, cross-sectional, descriptive study was conducted in the Adult Ward of the Botucatu University Hospital Emergency Unit - UNESP, Brazil. Data was collected by means of interviews, observation, and individual medical chart consultation, to be analyzed via logistic regression using the statistical software package SAS for Windows, version 9.2. The sample consisted of 71 subjects with a mean age of 74.30 years. It was observed that $30.99 \%$ of the older individuals showed to be independent in performing the proposed activities; $22.53 \%$ were partly dependent; and $46.48 \%$ were fully dependent. Gerontology care must aim at maintaining functional conditions, thus promoting independence and preserving these individuals' dignity.
\end{abstract}

DESCRIPTORS: Nursing. Older individuals' health. Geriatric assessment. Activities of daily living.

\section{EL GRADO DE DEPENDENCIA DE ANCIANOS HOSPITALIZADOS PARA LA REALIZACIÓN DE LAS ACTIVIDADES BÁSICAS DE LA VIDA DIARIA}

\begin{abstract}
RESUMEN: Este estudio pretende identificar el grado de dependencia de ancianos hospitalizados para la realización de las actividades básicas de la vida diaria. Se trata de un estudio cuantitativo, transversal y descriptivo, desarrollado en la Enfermería para Adultos del sector de Urgencias del Hospital das Clínicas de Botucatu - UNESP. La compilación de los datos ocurrió mediante entrevistas, observación y consulta de los expedientes individuales. El análisis de los datos se hizo con el paquete estadístico SAS for Windows versión 9.2, utilizando regresión logística. La muestra estuvo constituida por 71 sujetos, con edad media de 74,30 años. Se verificó que el 30,99\% de los ancianos fueron independientes en la realización de las actividades propuestas; el 22,53\%, parcialmente dependientes y el $46,48 \%$ totalmente dependientes. El cuidado en gerontología debe concentrarse en el mantenimiento del estado funcional, promover la independencia y preservar la dignidad de los sujetos.
\end{abstract}

DESCRIPTORES: Enfermería. Salud del anciano. Evaluación geriátrica. Actividades cotidianas. 


\section{INTRODUÇÃO}

O aumento da população idosa constitui tema de debate entre pesquisadores, gestores sociais e políticos de diversos países do mundo. ${ }^{1}$ Nas últimas décadas, temos assistido a um aumento significativo do número da população idosa mundial, com prognóstico de um crescimento ainda maior nos próximos anos. ${ }^{2}$

Conforme evidenciado por alguns estudos, ${ }^{1-3}$ a população brasileira também vem envelhecendo de forma rápida. O censo demográfico de 2000 apontou para uma população idosa de $14,5 \mathrm{mi}$ lhões de pessoas, o que corresponde a $8,6 \%$ da população total do País, estimando-se para o ano de 2020 a cifra de 30,9 milhões de pessoas idosas. Segundo projeções da Organização Mundial de Saúde (OMS), ${ }^{4}$ em 2025 o Brasil será o sexto país do mundo com maior número de idosos. Além disto, vale destacar que, dentro do contingente de idosos brasileiros, tem aumentado o número de pessoas com 80 anos ou mais, alterando a composição etária dentro do próprio segmento populacional idoso. ${ }^{2}$

Neste novo cenário populacional, a redução das taxas de fecundidade e de mortalidade eleva a expectativa de vida e remete à nova configuração populacional do País. Surge assim a transição epidemiológica, caracterizada pelo declínio das doenças infecto-parasitárias e aumento das enfermidades crônicas não transmissíveis. Dessa forma, a inversão da pirâmide populacional acarretará grandes transformações na estrutura sociopolítico-econômico-cultural da sociedade, e isso repercutirá diretamente no setor saúde, que irá requerer recursos, suporte e auxílio orientado por profissionais especializados. ${ }^{2}$

O envelhecimento é um processo comum a todos os seres vivos, sendo influenciado por múltiplos fatores (biológicos, psicológicos, econômicos, sociais, culturais, dentre outros). É um processo dinâmico e progressivo, no qual modificações morfológicas, funcionais e bioquímicas podem interferir na capacidade de adaptação do indivíduo ao meio social em que vive, tornando-o mais vulnerável aos agravos e doenças e comprometendo sua qualidade de vida. ${ }^{2}$

\section{Assistência de enfermagem ao paciente idoso}

Na assistência hospitalar, a idade é considerada um indicador na determinação da assistência ao idoso enfermo e o estado funcional é o parâmetro mais fidedigno no estabelecimento de critérios específicos de atendimento. A avaliação funcional é a mensuração da capacidade de uma pessoa de completar as tarefas funcionais e desempenhar as funções sociais, abordando especificamente a capacidade de completar tarefas que variam desde os simples cuidados pessoais até atividades de nível mais complexo. ${ }^{5}$

A capacidade funcional pode ser definida como sendo o grau de preservação do indivíduo na capacidade de realizar Atividades Básicas da Vida Diária (AVDS), como banhar-se, vestir-se, transferir, ter continência de esfincteres e alimentar-se, e também para desenvolver Atividades Instrumentais da Vida Diária (AIVDS) como cozinhar, arrumar a casa, telefonar, lavar roupa, ir às compras, cuidar das finanças domésticas e tomar remédios. ${ }^{6}$

A incapacidade funcional, ou disabilidade, definida pela dificuldade ou dependência do idoso na realização individual das atividades de vida diária, limita a autonomia do idoso, reduz sua qualidade de vida e aumenta o risco de dependência, institucionalização e morte prematura. A diminuição da capacidade funcional é referida também como fator de aumento no risco de quedas, principalmente devido ao comprometimento na realização de tarefas do dia a dia, com limitações de força muscular, equilíbrio e mobilidade; no Brasil, 30\% dos idosos caem pelo menos uma vez ao ano. A incapacidade funcional é um processo dinâmico e progressivo, consequência das doenças crônico-degenerativas e de mudanças fisiológicas associadas ao envelhecimento, podendo também ocorrer de forma aguda, como, por exemplo, no acidente vascular encefálico e na fratura de fêmur, que ocasionam limitações funcionais. ${ }^{7}$

A disabilidade é encontrada num percentual entre $20 \%$ e $30 \%$ dos idosos acima de 70 anos que vivem em comunidades nos Estados Unidos, aumentando com a idade. ${ }^{8}$ Estudos como o Established Populations for Epidemiologic Studies of the Elderly (EPESE), o Health interview survey longitudinal study on aging e o National long term care survey, verificaram que a incidência de incapacidade funcional aumenta com a idade e, quando tratada, há melhoria em idosos entre 65 e 74 anos, com menor severidade e duração desta incapacidade. ${ }^{8,9}$

As alterações biológicas tornam o idoso menos capaz de manter a homeostase quando submetido a um estresse fisiológico. Tais modificações, principalmente quando associadas à idade cronológica avançada, determinam maior suscetibilidade à ação de doenças, crescente vulnerabilidade e maior probabilidade de morte. A elevada 
prevalência de doenças crônico-degenerativas, somada à ocorrência de pluripatogenia (duas ou mais doenças concomitantes), têm sido consideradas responsáveis pela necessidade de maior permanência hospitalar e pela progressiva perda de autonomia dos idosos..$^{10}$ Desse modo, os idosos costumam apresentar necessidades peculiares. Um estudo ${ }^{3}$ que buscou identificar os principais diagnósticos de enfermagem em pacientes idosos hospitalizados, encontrou como resultados: risco para infecção $(100 \%)$, mobilidade física prejudicada $(50,7 \%)$, nutrição alterada: menos que as necessidades corpóreas $(44,7 \%)$, déficit no autocuidado $(43,3 \%)$ e integridade da pele prejudicada $(41,8 \%)$.

Durante a hospitalização, a capacidade funcional do idoso pode ser comprometida e levar à dependência funcional, por se tratar de um evento complexo que ocorre num momento de fragilidade, quando o idoso é retirado do seu meio e do convívio familiar e social, e transferido para um ambiente estranho. ${ }^{6,11}$ Atualmente, tem crescido o número de estudos que enfocam a tríade envelhecimento, capacidade funcional e hospitalização. A literatura comprova que o declínio funcional acomete de 34 a $50 \%$ dos idosos durante o período de hospitalização. Contudo, ainda não se sabe ao certo até onde esse comprometimento é secundário apenas ao processo de hospitalização ou influenciado por fatores como gravidade da doença, estado nutricional, terapêutica empregada e o ambiente não responsivo. ${ }^{6,12}$

A avaliação funcional é essencial no tratamento do idoso, uma vez que as condições crônico-degenerativas, tão frequentes nessa faixa etária, tendem a afetar a capacidade funcional dos idosos. ${ }^{13}$ Idosos classificados como totalmente dependentes constituem um grupo geralmente enquadrado como mais vulnerável. Os serviços de saúde deverão estar preparados para identificá-los e promover-lhes assistência diferenciada e pautada na participação de diferentes profissionais da área da saúde. ${ }^{1}$ Sendo assim, torna-se importante perceber que são normais algumas perdas e dificuldades que o idoso apresenta, bem como saber diferenciar um processo normal de um patológico. Tal discernimento permite subsidiar o planejamento da assistência profissional (Sistematização da Assistência de Enfermagem /SAE), que também deve estar em sintonia com o contexto familiar do idoso, considerando as nuances sociais e culturais presentes nessa relação. ${ }^{2,3}$

Observa-se que existem dados parciais e inconclusivos quanto ao comprometimento funcional do idoso que é hospitalizado. Somado a isso, o alto número de óbitos dentre essa população dificulta o acompanhamento desses idosos, desestimulando a realização de estudos longitudinais que poderiam esclarecer várias incertezas existentes. ${ }^{6}$

Estudos realizados em pacientes hospitalizados, ambulatoriais e em reabilitação, concluíram que a avaliação funcional pode detectar deficiências importantes no desempenho funcional que podem se ocultar durante os exames clínicos convencionais. Durante a internação, a avaliação funcional fornece dados que são importantes no prognóstico e proporcionam uma linguagem comum, entre os profissionais da saúde, além de facilitar a pesquisa epidemiológica. ${ }^{6}$ Desta forma, justifica-se a realização deste estudo, que objetivou traçar o perfil socioeconômico e identificar o grau de dependência de idosos hospitalizados na realização das Atividades Básicas da Vida Diária (AVDs).

\section{MÉTODO}

Trata-se de estudo quantitativo, transversal e descritivo, realizado na Enfermaria de Adultos do Serviço Técnico de Pronto-Socorro, do Hospital das Clínicas da Faculdade de Medicina de Botucatu - UNESP, caracterizado como um hospital geral universitário.

A coleta de dados ocorreu no período de maio a outubro de 2009, por meio de entrevistas, observação e registro de informações constantes nos prontuários, utilizando-se um instrumento de coleta de dados elaborado especificamente para este estudo. Não foi feito cálculo amostral, e sim, uma amostra de conveniência, uma vez que foram incluídos no estudo todos os idosos que permaneceram internados por, no mínimo, 48 horas, na enfermaria em questão, no período de tempo referido.

O tempo de 48 horas mínimas de internação foi estabelecido para que houvesse tempo suficiente para a pesquisadora apreender as necessidades de auxílio apresentadas pelos pacientes, consultar prontuários e entrevistar os familiares, nos horários de visita, no caso dos idosos que não podiam responder. Consideramos como idosas, neste estudo, as pessoas com idade igual ou superior a 60 anos, conforme estabelecido pela Lei $\mathrm{N}^{\mathrm{o}} 8.842 / 1994 .{ }^{1}$

A pesquisadora frequentou diariamente a enfermaria, permanecendo duas horas por dia (tempo do horário de visitas), identificando os sujeitos da pesquisa para entrevista e observação e registrando os dados no instrumento elaborado 
para este fim. Os idosos foram entrevistados na primeira visita da pesquisadora após a admissão do paciente na enfermaria. Nas situações em que a entrevista com o paciente idoso se apresentou inviabilizada, entrevistou-se o familiar responsável pelo paciente, ressaltando que os familiares só foram entrevistados nos casos em que o idoso se apresentava impossibilitado de responder (processo demencial, estado de inconsciência, dificuldade para verbalizar). A família, quando estava presente, permanecia com o paciente durante todo o período de visitas, mas só era entrevistada nestes casos citados acima. Foram excluídos do estudo os idosos que não podiam responder e cujos familiares não foram localizados pela pesquisadora durante o período de internação do idoso.

$\mathrm{Na}$ entrevista foram perguntados apenas itens referentes à idade, estado civil, número de filhos, anos de estudo e ocupação que exercia ou exerce. As demais informações foram obtidas a partir da observação do paciente no leito e de informações contidas nos prontuários individuais: tipo de banho do paciente (banho de leito ou de aspersão), necessidade de auxílio para o banho, para vestir-se, para locomoção e para alimentação, uso do toalete, uso de fraldas, presença de sondas enterais e vesicais.

A avaliação do grau de dependência foi realizada de acordo com o Índex de Katz, ${ }^{14}$ um dos instrumentos mais antigos e também dos mais citados na literatura para avaliação funcional de idosos. O instrumento proposto por Katz avalia o grau de dependência para as Atividades Básicas da Vida Diária (AVDs) e para as Atividades Instrumentais da Vida Diária (AIVDs), com base na necessidade ou não de auxílio para realização das tarefas. ${ }^{13}$ No caso do presente estudo, devido às peculiaridades da internação hospitalar, que impossibilitam a avaliação da maioria das AIVDs, a avaliação funcional dos idosos está voltada para as AVDs.

Para avaliação do grau de dependência relacionado às AVDs, abordamos as seis atividades propostas por Katz: banho, vestir-se, uso do toalete, transferência, continência de esfincteres e alimentação. O grau de dependência, neste estudo, se apresenta como uma variável politômica com três categorias (independente, parcialmente dependente e totalmente dependente). Para classificar os sujeitos de acordo com as atividades propostas por Katz, atribuiu-se uma nota para cada uma das AVDs, sendo que os indivíduos que se apresentaram independentes na realização da tarefa receberam 0 ponto e os que apresentavam dependência receberam um ponto. Desse modo, os idosos que obtiveram escore final de zero ponto foram classificados como independentes, os que obtiveram escore de seis como totalmente dependentes e os que apresentaram escore de um a cinco como parcialmente dependentes.

Os dados foram organizados e codificados manualmente no instrumento de coleta, com objetivo de reduzir possíveis erros na identificação das respostas e de diminuir o tempo despendido na digitação do banco de dados. Após essa etapa, os dados foram digitados em planilha do programa Microsoft Office Excel 2003® e posteriormente analisados estatisticamente pelo programa $S A S$ for Windows, versão 9.2®. Todos os dados foram coletados, registrados manualmente e digitados por uma única pesquisadora. As coautoras realizaram a revisão do banco de dados, visando ao controle de qualidade da digitação.

Para as variáveis categorizadas, foram estabelecidas freqUências absolutas e porcentagens e para as variáveis quantitativas, média, desvio-padrão, mediana, valor máximo e valor mínimo. Considerando-se o grau de dependência dos sujeitos como a variável desfecho e "idade", em anos, e "tempo de internação", em dias, como variáveis explanatórias, foi ajustado um modelo de regressão logística para dados ordinais de chances proporcionais (já que as categorias são dependentes), com a obtenção do Odds Ratio (OR) e dos respectivos intervalos de confiança de $95 \%$. Para o ajuste deste modelo foi utilizada a PROC LOGISTIC do programa SAS for Windows, versão 9.2. Em todos os testes, considerou-se o nível de significância de 5\%.

O projeto de pesquisa foi aprovado pelo Comitê de Ética em Pesquisa da Faculdade de Medicina de Botucatu (Ofício 14/09-CEP), conforme Resolução CNS 196/96. Os sujeitos do estudo e/ou seus responsáveis foram devidamente esclarecidos sobre os objetivos e métodos da pesquisa e só foram entrevistados e incluídos no estudo após assinarem o Termo de Consentimento Livre e Esclarecido.

\section{RESULTADOS}

Estiveram internadas na Enfermaria de Adultos do Pronto-Socorro do HC - UNESP, no período do estudo, 113 pessoas com idade igual ou superior a 60 anos, o que representa $52,31 \%$ do total de internações (216).

A amostra deste estudo foi constituída por 71 sujeitos, o que corresponde a $62,83 \%$ dos idosos internados na enfermaria no período em questão. Os demais indivíduos idosos (42) não foram incluídos 
por apresentarem tempo de internação inferior a 48 horas (conforme critérios de inclusão do estudo), com exceção de oito indivíduos que apresentaram tempo de internação igual ou superior a 48 horas, mas não foram incluídos por não terem condições de responder às perguntas e assinar o Termo de Consentimento Livre e Esclarecido e os familiares não terem sido encontrados pela pesquisadora durante o período de internação. Em 53,52\% dos idoso, nos $46,48 \%$ restantes o entrevistado foi o familiar responsável (filho, cônjuge, irmão).

\section{Perfil socioeconômico da amostra}

Quanto ao sexo, 36 indivíduos (50,70\%) pertencem ao sexo masculino e $35(49,30 \%)$ ao sexo feminino. A média de idade dos sujeitos é de 74,30 anos (desvio padrão de 9,48 e mediana de 74 anos), sendo a idade mínima encontrada de 60 anos e a máxima de 107 anos. A maioria dos idosos $(73,24 \%)$ possui idade entre 60 e 79 anos, de modo que $38,03 \%$ pertencem à faixa etária de 60 a 69 anos e $35,21 \%$ à faixa de 70 a 79 anos.

Para os indivíduos do sexo masculino, a média de idade foi de 72,72 anos (desvio padrão de 7,86, mediana de 73,50, valor mínimo de 61 anos e máximo de 92 anos), já para o sexo feminino a média foi de 75,91 anos (desvio padrão de 10,77, mediana de 77, valor mínimo de 60 anos e máximo de 107 anos).

O número médio de anos de estudo da amostra é de 2,57 anos (desvio padrão de 2,98 e mediana de três anos), sendo quatro sujeitos $(5,63 \%)$ excluídos deste cálculo devido ao fato de o familiar entrevistado não ter conhecimento do número de anos de estudo do idoso. O número máximo de anos de estudo encontrado foi de 15 anos e o mínimo de zero, sendo que 28 sujeitos (41,79\%) não possuem nenhum ano de estudo. Dentre estes 28 indivíduos, $16(57,14 \%)$ foram alfabetizados em casa e sabiam, pelo menos, escrever o próprio nome; os demais eram analfabetos.

Quanto à profissão/ocupação que os idosos exercem ou exerciam, as mais frequentes foram: trabalhador rural (26,76\%), dona de casa $(16,90 \%)$, empregada doméstica $(12,68 \%)$ e pedreiro $(7,04 \%)$. Outras profissões/ocupações citadas foram: operador de máquinas, caminhoneiro, costureira, metalúrgico, motorista, assistente social, profissional autônomo, auxiliar de serviços gerais, balconista, corretor de imóveis, cozinheira, empresário, operador de sistemas elétricos, segurança, torneiro mecânico, técnico em administração e técnico em laboratório. Um casos (38 indivíduos) o entrevistado foi o próprio

indivíduo $(1,41 \%)$ não teve definição de profissão porque a familiar entrevistada não soube informar a profissão que o idoso havia exercido.

Com relação ao estado civil, 28 idosos $(39,44 \%)$ encontravam-se casados, $28(39,44 \%)$ viúvos, seis $(8,45 \%)$ solteiros, cinco $(7,04 \%)$ divorciados e quatro $(5,63 \%)$ vivendo em união estável com o parceiro. Considerando-se o estado civil em relação ao sexo, encontraram-se oito pessoas do sexo feminino casadas, duas divorciadas, três solteiras, duas em união estável e 20 viúvas. No sexo masculino encontraram-se 20 indivíduos casados, três divorciados, três solteiros, dois em união estável e oito viúvos.

O número médio de filhos por indivíduo é de 3,65 (desvio padrão de 2,69 e mediana de 3,00 filhos), o número mínimo encontrado foi de nenhum filho (zero) e o maior de 13 filhos. A maioria das pessoas $(56,40 \%)$ possui entre um e quatro filhos (Tabela 1 ).

Tabela 1 - Distribuição de frequências das variáveis sociodemográficas da amostra de idosos internados na enfermaria de adultos do ProntoSocorro do Hospital das Clínicas da Faculdade de Medicina de Botucatu no período de maio a outubro de 2009

\begin{tabular}{|c|c|c|}
\hline Variáveis & $\mathbf{n}$ & $\%$ \\
\hline \multicolumn{3}{|l|}{ Sexo (71) } \\
\hline Masculino & 36 & 50,70 \\
\hline Feminino & 35 & 49,30 \\
\hline \multicolumn{3}{|c|}{ Faixa etária (71) } \\
\hline 60 a 69 anos & 27 & 38,03 \\
\hline 70 a 79 anos & 25 & 35,21 \\
\hline 80 a 89 anos & 13 & 18,31 \\
\hline 90 a 99 anos & 5 & 7,04 \\
\hline 100 a 110 anos & 1 & 1,41 \\
\hline \multicolumn{3}{|c|}{ Anos de estudo $(67)^{*}$} \\
\hline Zero & 28 & 41,79 \\
\hline 1 a 4 & 34 & 50,75 \\
\hline 5 a 8 & 3 & 4,48 \\
\hline 9 ou mais & 2 & 2,99 \\
\hline \multicolumn{3}{|c|}{ Estado civil (71) } \\
\hline Casado & 28 & 39,44 \\
\hline Divorciado & 5 & 7,04 \\
\hline Solteiro & 6 & 8,45 \\
\hline União estável & 4 & 5,63 \\
\hline Viúvo & 28 & 39,44 \\
\hline \multicolumn{3}{|c|}{ Número de filhos (71) } \\
\hline Zero & 9 & 12,68 \\
\hline 1 a 4 & 40 & 56,34 \\
\hline 5 a 8 & 18 & 25,35 \\
\hline 9 ou mais & 4 & 5,63 \\
\hline
\end{tabular}

Texto Contexto Enferm, Florianópolis, 2011 Jan-Mar; 20(1): 59-67. 


\section{Avaliação funcional}

Na avaliação do grau de dependência da equipe de enfermagem para realização das Atividades Básicas da Vida Diária, 22 idosos (30,99\%) apresentaram-se independentes (0 ponto), ou seja, realizavam todas as seis AVDs sem auxílio; $16(22,53 \%)$ apresentaram-se parcialmente dependentes (1 a 5 pontos), realizando sozinhos apenas algumas das AVDs e 33 idosos (46,48\%) mostraram-se totalmente dependentes (6 pontos), ou seja, não realizavam nenhuma das AVDs sem auxílio, necessitando, portanto, de banho no leito ou banho de aspersão com auxílio na cadeira de rodas; alimentação por meio de sonda nasoenteral ou por via oral com a assistência do profissional de saúde; uso de sonda vesical de demora, fraldas, comadres e papagaios; transferência mecânica realizada pelos profissionais, dentre outras necessidades.

Dentre os 16 pacientes considerados como parcialmente dependentes, quatro dependiam da equipe de enfermagem apenas para a AVD
Continência de esfíncteres (1 ponto), dois dependiam apenas para a AVD Alimentação (1 ponto), 2 dependiam da equipe para as AVDs Uso do toalete, Banho e Movimentação (3 pontos), e a maioria (oito pacientes) dependia da equipe de enfermagem para realizar todas as AVDs, com exceção da Alimentação (5 pontos).

A idade dos sujeitos e o grau de dependência não se mostraram estatisticamente relacionados, ou seja, os indivíduos com mais idade não se mostraram mais dependentes do que os com menos idade e vice-versa $(p=0,4751)$. Contudo, o grau de dependência esteve relacionado ao tempo de internação $(p=0,0467)$, conforme consta na tabela 2. Como o tempo de internação foi considerado como uma variável contínua e obteve-se $\mathrm{OR}=1,078$, com IC variando entre 1,001 e 1,162, tem-se que o risco do indivíduo passar de independente para parcialmente dependente e de parcialmente dependente para totalmente dependente aumenta em $7,8 \%$ (variando de $1 \%$ a $16 \%$ ) a cada dia de internação.

Tabela 2 - Ajuste do modelo de regressão logística entre o grau de dependência e as variáveis idade e tempo de internação da amostra de idosos internados na enfermaria de adultos do Pronto-Socorro do Hospital das Clínicas da Faculdade de Medicina de Botucatu no período de maio a outubro de 2009

\begin{tabular}{lcccc}
\hline Variável & Estimativa & Desvio padrão & OR (IC 95\%) & p-valor \\
\hline Idade & 0,0173 & 0,0243 & $1,017(0,970-1,067)$ & 0,4751 \\
Tempo de internação & 0,0755 & 0,0379 & $1,078(1,001-1,162)$ & 0,0467 \\
\hline
\end{tabular}

Sete dos idosos $(9,86 \%)$ já eram previamente acamados, sendo totalmente dependentes dos cuidados por parte dos familiares ou pessoas responsáveis. Quanto ao tempo de internação dos idosos na Enfermaria de Adultos doSTPS, a média foi de 8,35 dias (desvio padrão de 7,89 e mediana de seis dias), de modo que o tempo mínimo encontrado foi de dois dias (conforme critérios do estudo) e o máximo foi de 46 dias.

Com relação ao destino do paciente ao sair da enfermaria, 49 pessoas $(69,01 \%)$ receberam alta hospitalar, 11 pacientes $(15,49 \%)$ foram a óbito e os outros $11(15,49 \%)$ foram transferidos para outras unidades de internação do HC-UNESP, sendo que quatro deles foram transferidos para Unidades de Terapia Intensiva (UTI) devido à piora do estado geral.

\section{DISCUSSÃO}

Corroborando os censos e projeções que têm indicado um crescimento cada vez maior do segmento idoso da população, ${ }^{1-3}$ os idosos representaram, neste estudo, 52,31\% do total de internações da Enfermaria de Adultos do ProntoSocorro. Houve predomínio do sexo feminino, assim como em outros estudos realizados com idosos hospitalizados, ${ }^{11,15}$

Houve predominância de idosos com idade inferior a 79 anos, dado condizente com outro estudo realizado no Brasil com idosos hospitalizados. ${ }^{10}$ Os idosos do sexo masculino apresentaram uma média de idade inferior aos dos indivíduos do sexo feminino, o que talvez se justifique pelo fato de as mulheres possuírem uma expectativa de vida maior que a dos homens. ${ }^{2}$ Segundo o Anuário Estatístico de Saúde do Brasil, no que se refere à esperança de vida ao nascer, por sexo, há uma diferença de quase oito anos, sendo de 64,8 anos para os homens e 72,6 anos para as mulheres. ${ }^{16}$

A baixa escolaridade encontrada condiz com um estudo realizado com idosos internados em enfermaria de um hospital geral, ${ }_{15}^{15}$ cujos resultados apontaram que $45,71 \%$ dos idosos possuíam 
baixa escolaridade, $42,86 \%$ eram analfabetos e $11,43 \%$ apresentavam média escolaridade. O baixo grau de instrução, quando associado às condições econômicas precárias e à dificuldade de acesso à rede de suporte, pode trazer dificuldades no autocuidado e no gerenciamento dos problemas pelo idoso e por sua família, acarretando a institucionalização. ${ }^{5}$

A baixa escolaridade também se mostra associada às profissões/ocupações referidas, ou seja, encontramos predominantemente profissões que não requerem alto nível de escolarização como: trabalhador rural, empregada doméstica, pedreiro, entre outras. Encontraram-se apenas dois sujeitos com escolaridade igual ou superior a nove anos de estudo, que exerciam as profissões de Técnico em Administração e Técnico de Laboratório.

Predominaram os idosos casados e os viúvos. Percebe-se que o número de solteiros, divorciados e em união estável foi pequeno e igual ou muito parecido nos dois sexos; entretanto encontrou-se um número bem maior de indivíduos casados no sexo masculino e um número bem maior de viúvos no sexo feminino. Um estudo ${ }^{17}$ aponta que a predominância da viuvez para o sexo feminino ocorre por causa da alta expectativa de vida feminina e porque as mulheres, ao se tornarem viúvas, tendem a permanecer nessa situação. Os homens, por sua vez, ao perderem as parceiras, têm a tendência de se casar novamente.

O número médio de filhos por indivíduo foi de 3,65. Nas últimas décadas, tem ocorrido, cada vez mais, um declínio nas taxas de fecundidade. Os dados demográficos têm demonstrado que as famílias geram cada vez menos filhos, fato que, num futuro próximo, poderá constituir um fator dificultador para o cuidado familiar ao idoso. ${ }^{5}$

$\mathrm{Na}$ avaliação do grau de dependência da equipe de enfermagem para realização das AVDs, predominaram os idosos totalmente dependentes, o que reflete um grande aumento do tempo do cuidado de enfermagem. Mesmo entre os idosos considerados parcialmente dependentes, percebese que a maioria deles dependeu da equipe de enfermagem para realizar todas as AVDs, com exceção apenas da alimentação. Os dados são condizentes com outros trabalhos realizados com idosos hospitalizados, sendo que um dos estudos ${ }^{6}$ mostrou que aproximadamente $49 \%$ da amostra apresentava incapacidade para sete ou mais tarefas, e o outro ${ }^{15}$ encontrou que $42,85 \%$ dos idosos possuíam alta independência, 34,28\% média independência e $22,85 \%$ alta dependência.
A literatura aponta que ocorrem quase duas vezes mais perdas funcionais para as Atividades Instrumentais do que para as Básicas, sendo sugestivo que as AIVDs possam ser marcadores sensíveis do grau de socialização da pessoa idosa. Desse modo, quando o idoso apresenta inabilidade para alguma das AVDs, provavelmente também já apresenta inabilidade para duas ou mais AIVDs. ${ }^{5,18}$

A dependência não é um estado permanente, mas sim um processo dinâmico, cuja evolução pode ser modificada, prevenida e/ou reduzida. Tal evolução nesse processo sugere a existência de profissionais qualificados e comprometidos com a assistência ao idoso. As necessidades de cuidado requerem desenvolvimento de suas atividades básicas da vida diária. O elevado grau de dependência requer uma equipe de enfermagem adequada, tanto quantitativa, quanto qualitativamente. As unidades de internação para idosos necessitam de um número diferenciado de profissionais de enfermagem, uma vez que estes pacientes apresentam uma dependência grande da equipe para realização de atividades básicas. Além disso, a equipe deve estar capacitada para lidar com situações específicas do cuidado do idoso, respeitando a dependência, mas incentivando a autonomia.

Neste estudo, a idade dos sujeitos e o grau de dependência dos mesmos não se mostraram estatisticamente relacionados, ou seja, os indivíduos de maior idade não se mostraram mais dependentes que os de menor idade, demonstrando que, muitas vezes, o surgimento das incapacidades é decorrente de características individuais (como patologias associadas, herança genética, hábitos de vida) e não do fator idade em si. Este dado está de acordo com um estudo ${ }^{11}$ realizado em São Paulo com idosos hospitalizados, mas contradiz alguns estudos estrangeiros. ${ }^{19-21}$

O tempo de internação dos idosos na enfermaria em questão foi, em média, de 8,35 dias, o que representa uma média bastante alta para uma enfermaria de Pronto-Socorro. Não foram encontrados outros estudos com o tempo médio de internação em enfermaria de Pronto- Socorro, mas na maioria dos estudos estrangeiros, a variação é de oito a 15 dias de hospitalização em unidades gerais de internação. ${ }^{11,20,21}$ Vale lembrar que este valor provavelmente não expressa exatamente a realidade do serviço estudado, uma vez que estamos trabalhando neste estudo apenas com os idosos com tempo de internação igual ou superior a 48 horas. Contudo, esta média ainda é capaz de revelar o 
problema de organização de fluxo vivenciado pela instituição, sendo que muitas vezes não há leitos nas enfermarias de internação das especialidades e o paciente permanece durante todo o seu período de internação na enfermaria do Pronto-Socorro, unidade esta que não apresenta estrutura física adequada para internações de longo período.

Um estudo multicêntrico internacional revela que a capacidade funcional de idosos internados em unidades de Pronto-Socorro, como é o caso do presente estudo, é subavaliada e subjugada, sendo que os médicos geralmente não fazem referência ao estado funcional do paciente no prontuário e nem sempre levam este fator em conta nas tomadas de decisão clínica. ${ }^{22}$

As limitações do presente estudo são referentes à amostra pouco numerosa de idosos, ao curto período de coleta de dados e ao fato de não relacionar o grau de dependência com outras variáveis, como estado nutricional e comorbidades apresentadas.

\section{CONSIDERAÇÕES FINAIS}

O envelhecimento, principalmente com dependência, é um desafio a ser incorporado pela Saúde Pública. A saúde não é medida pela presença ou não de doenças, e sim pelo grau de preservação da capacidade funcional. Um dos desafios complexos da atualidade acadêmica e profissional é o referente à motivação para aprender a trabalhar de forma interacional e integrada na prevenção e tratamento da incapacidade funcional em idosos.

Esta pesquisa contribuiu para a estruturação de assistência de enfermagem de melhor qualidade a ser prestada aos idosos, uma vez que colabora para o conhecimento de características desta clientela, como o perfil sociodemográfico e o seu nível de dependência para realização das AVDs durante a hospitalização. Os resultados desta investigação contribuem também para reflexão acerca do manejo do processo saúde doença em todos os níveis de atenção à saúde, destacando a prioridade que se deveria dar à promoção da saúde e prevenção de agravos.

Urge que o cuidado integralizado seja uma realidade no atendimento ao idoso. Portanto, é necessário respeitar a velhice e seu processo; utilizar uma abordagem individual centrada na pessoa, e não na doença; considerar o idoso como participante ativo no controle e no tratamento da saúde e lutar por condições cada vez mais humanas da assistência. A especificidade quanto à população idosa está em atentar para suas vulnerabilidades, manutenção de sua capacidade funcional, sua autonomia e minimização de sua dependência.

A enfermagem gerontológica, no Brasil, ainda carece de profissionais que atuem nessa área especializada. Essa área focaliza a prestação de cuidados ao idoso pela enfermagem, sendo consideradas as necessidades e as características como únicas, o que promoverá um cuidado integral e contextualizado para essa população. Uma das principais responsabilidades do enfermeiro geriátrico é compreender o processo do envelhecimento como natural e não como patológico, devendo estabelecer uma relação terapêutica com o usuário/idoso.

Além de ressaltar o dilema da incapacidade funcional no cotidiano do idoso, devemos refletir também sobre as implicações da disabilidade para o cuidado de enfermagem do idoso, hospitalizado. Muitas vezes as unidades de internação não contam com um número adequado de profissionais e estes não são capacitados para o cuidado específico do paciente idoso principalmente em se tratando de uma enfermaria de Pronto-Socorro, como é o caso deste estudo. Desse modo, a incapacidade funcional deve ser sempre levada em conta no planejamento estrutural da unidade e no planejamento de cuidados, incluindo tanto o dimensionamento de pessoal, quanto a capacitação e suporte oferecido à equipe para realizar um cuidado humanizado e voltado para a autonomia do paciente.

Sugerimos que novos estudos sejam realizados, na busca de maior clareza sobre a problemática da incapacidade funcional do idoso hospitalizado, com amostras mais numerosas e variadas de idosos em acompanhamento longitudinal e com a análise de outras variáveis como tipo e gravidade das doenças, estado nutricional, terapêutica empregada, interferências ambientais, entre outros. Sugerimos também que a administração hospitalar e os profissionais envolvidos na assistência ao idoso sejam conscientizados da vulnerabilidade funcional do idoso hospitalizado, para que medidas administrativas e assistenciais sejam adotadas com o intuito de minimizar, ao máximo, o declínio funcional nesse processo e, com isso, contribuir para o envelhecimento saudável.

\section{REFERÊNCIAS}

1. Rodrigues RAP, Kusumota L, Marques S, Fabrício SCC, Cruz IR, Lange C. Política nacional de atenção ao idoso e a contribuição da enfermagem. Texto Contexto Enferm. 2007 Jul-Set; 16(3):536-45. 
2. Souza RF, Skubs T, Brêtas ACP. Envelhecimento e família: uma nova perspectiva para o cuidado de enfermagem. Rev Bras Enferm. 2007 Mai-Jun; 60(3):263-7.

3. Sakano LM, Yoshitome AY. Diagnósticos e intervenções de enfermagem em idosos hospitalizados. Acta Paul Enferm. 2007 Out-Dez; 20(4):495-8.

4. Organização Mundial de Saúde. The world health report 2001. Geneva: WHO; 2001.

5. Aires M,Paz AA, Perosa CT. Ograu de dependência e características de pessoas idosas institucionalizadas. RBCEH. 2006 Jul-Dez 3(2):79-91.

6. Kawasaki K, Diogo MJD. Variação da independência funcional em idosos hospitalizados relacionada à variáveis sociais e de saúde. Acta Fisiatr. 2007 Set; 14(3): 164-9.

7. Bonardi G, Azevedo e Souza VB, Moraes JFD. Incapacidade funcional e idosos: um desafio para os profissionais de saúde. Sci. med. 2007 Jul-Set; 17(3):138-44

8. Fried LP, Guralnik JM. Disability in older adults: evidence regarding significance, etiology, and risk. J Am Geriatr Soc. 1997 Jan; 45(1):92-100.

9. Fried LP, Tangen CM, Walston J, Newman AB, Hirsch C, Gottdiener J, et al. Frailty in older adults: evidence for a phenotype. J.Gerontol. 2001; 56: M146-56.

10. Sales FM, Santos I. Perfil de idosos hospitalizados e nível de dependência de enfermagem: identificação de necessidades. Texto Contexto Enferm. 2007 JulSet; 16(3):495-502.

11. Siqueira AB, Cordeiro RC, Perracini MR, Ramos LR. Impacto funcional da internação hospitalar de pacientes idosos. Rev. Saúde Pública. 2004 Out; 38(5):687-94.

12. Kamper AM, Stott DJ, Hyland M, Murray HM, Ford I. Predictor of funcional decline in elderly people with vacular risk factors or disease. Age Ageing. 2005 Jul; 34: 450-5.

13. Duarte YAO, Andrade CL, Lebrão ML. O índice de Katz na avaliação da funcionalidade dos idosos. Rev Esc Enferm USP. 2007 Jun; 41(2):317-25.
14. Katz S, Downs TD, Cash HR, Grotz RC. Progress in development of the index of ADL. Gerontologist. 1970 Jan; 10(1):20-30.

15. Izzo H, Gruner T. Impacto da internação hospitalar na capacidade funcional do idoso em enfermaria geriátrica. Mundo Saúde. 2005 Out-Dez; 29(4):629-38.

16. Ministério da Saúde. Anuário estatístico de saúde do Brasil 2001 [Internet]. Brasília: Ministério da Saúde; 2002 [acesso 2008 Out 25]. Disponível em: http://portal. saude.gov.br/saude/ aplicacoes/ anuario2001/index.cfm

17. Camarano AA. Envelhecimento da população brasileira: uma contribuição demográfica. In: Freitas EV, Py L, Néri AL, Cançado FAX, Gorzoni ML, Rocha SM, organizadores. Tratado de geriatria e gerontologia. Rio de Janeiro (RJ): Guanabara Koogan; 2002. p. 58-71.

18. Maciel ACC, Guerra RR. Influência dos fatores biopsicossociais sobre a capacidade funcional de idosos residentes no nordeste do Brasil. Rev Bras Epidemiol. 2007 Jun; 10(2):178-89.

19. Creditor MC. Hazards of hospitalization of the elderly. Ann Inter Med. 1993 Feb; 118(3):219-23.

20. Hirsch CH, Sommers L, Olsen A, Muller L, Winograg $\mathrm{CH}$. The natural history of functional morbidity in hospitalized older patients. J Am Geriatr Soc. 1990 Dec; 38(12): 1296-303.

21. Inouye SK, Peduzzi PN, Robison JT, Hughes JS, Horwitz RI, Concato J. Importance of functional measures in predcting mortality among older hospitalized patients. JAMA 1998 apr; 279(15):1187-93.

22. Rodríguez-Molinero A, López-Diéguez M, Tabuenca AI, de la Cruz JJ, Banegas JR. Functional assessment of older patients in the emergency department: comparison between standard instruments, medical records and physicians' perceptions. BMC Geriatr [online] 2006 sept [Cited 2010 Jun 14]; 6: 13. Available from: http://www.ncbi.nlm.nih.gov/ pmc/ articles/PMC1569831/?tool=pubmed 